\title{
Modernized Solar Radio Spectrograph in the L Band Based on Software Defined Radio
}

\author{
Pavel Puricer ${ }^{1, *\left(\mathbb{D}, \text { Pavel Kovar }^{1}(\mathbb{D} \text { and Miroslav Barta }\right.}{ }^{2, *(\mathbb{D})}$ \\ 1 Department of Radioelectronics, Faculty of Electrical Engineering, Czech Technical University, Technicka 2, \\ 16627 Prague, Czech Republic \\ 2 Astronomical Institute, Czech Academy of Sciences, 25165 Ondrejov, Czech Republic \\ * $\quad$ Correspondence: puricep@fel.cvut.cz (P.P.); barta@asu.cas.cz (M.B.); Tel.: +420-22-435-5969 (P.P.)
}

Received: 28 June 2019; Accepted: 1 August 2019; Published: 3 August 2019

\begin{abstract}
The paper presents the concept, implementation, and test operation of a modernized solar radio spectrograph for an investigation of the solar emission and solar bursts in radio frequency bands. Besides having a strong diagnostic significance for studying the flare energy release, the solar radio bursts can also cause strong interference for radio communication and navigation systems. The current spectrograph for the Ondrejov observatory (Astronomical Institute of Czech Academy of Sciences) was modernized by using a direct-conversion receiver connected to a field-programmable gate array (FPGA) for the fast Fourier transform (FFT) spectrum estimation and put into the test operation. The higher time and frequency resolution and lower noise in comparison with the existing analog instrument were reached by the implementation of the latest optimal signal processing methods. To reduce the costs for such modernization, the operating frequency range was divided into four sub-bands of bandwidth $250 \mathrm{MHz}$, which brings another benefit of greater scalability. The first observations obtained by the new spectrograph and their comparison with the analog device are presented in the paper with future steps to put the spectrograph into the regular operation.
\end{abstract}

Keywords: spectrograph; FPGA; direct conversion receiver; software defined radio

\section{Introduction}

\subsection{Radio Diagnostics in Solar Flare Research}

Solar eruptions and flares represent the most powerful energy release processes in the entire solar system. Disturbances connected with these phenomena (magnetic clouds, beams of accelerated particles, enhanced radiation-namely in X-ray, UV, and radio bands) propagating from the Sun through the interplanetary space, are now known as effects of the space weather. In the case of reaching the Earth and its vicinity, they represent a serious risk for technologies (e.g., enhanced geo-currents-a risk for power distribution grids, ionospheric/TEC disturbances-an impact to the navigation systems and communications [1]) and also for humans (astronauts in the open space, aircraft crews and passengers for near-pole flights, etc.). In order to be able to give deterministic predictions of the space weather, we need to understand its drivers first. As the eruptive flares belong to those most effective, a research in the solar-flare physics represents a key to the future space weather forecasts.

Among all the radiation produced by solar flares the radio emission in decimetric range is the most closely related to the plasma processes of the flare energy release. Indeed, a radio emission connected with the beams of particles accelerated during the flare via a magnetic reconnection represents the first "messenger" of the solar flare ever [2]. Radio spectrographs, which are capable to record the dynamics of spectra on very short time scales, thus represent a key tool for remote radio diagnostics of the processes that started the flare. Modern approaches to the flare energy release via a magnetic 
reconnection [3] involve the energy cascading and fragmentation of the magnetic field structures in the solar atmosphere towards small scales. The small-scale structures, which are expected (as indicated by our numerical simulations) to be the actual locations of an energy release in flares, have also short dynamic timescales (millisecond and shorter). In order to be able to observe their radio signatures, the radio spectrograph should have a comparable temporal resolution. This represents a very strong driver for the spectrograph upgrade from the point of view of the astrophysical research.

\subsection{Direct Influence of the Solar Radio Bursts on the Radio Systems}

In addition to its important diagnostic role in the solar flare research, the solar radio emission (especially high-energy solar radio bursts) has a big impact on the navigation, communication, mobile, and Internet of things (IoT) systems and in a wider consequence to current and future information and transportation systems as well [4-9]. The influence of solar emissions can be observed as an increase of the effective noise temperature (or relevant noise factor) of the receiver as described in [10].The statistics of the solar radio bursts was published for example in [11] using a Poisson distribution for a description of the solar radio burst events together with an estimation of the distribution parameters from the long-term observation data. It can serve as a source for an estimation of the probability of high intensity events and their possible threats to the radio systems.

The influence of a solar radio burst to the popular satellite navigation system GPS (global positioning system) was documented in [4,6,7,12]. The unique radio burst from December 2006 with the peak level of one million SFU (solar flux unit) caused a significant reduction of the signal to noise ratio of the most GPS receivers, some receivers had lost tracking of the satellites and the acquisition of the new satellites was not possible due to the higher noise background. However, the performance degradation can occur for lower levels of radio bursts as well, for example a solar radio burst with the intensity 100,000 SFU causes in a common GPS receiver with a typical noise temperature $150 \mathrm{~K}$ and antenna gain $3 \mathrm{~dB}$ a drop of signal to noise ratio (SNR) by $15 \mathrm{~dB}$ that can prevent the receiver to acquire new signals [13].

The performance degradation can be also expected for the satellite-based augmentation systems (SBAS) [6]. These systems were developed as an improvement of the precision and safety of the current satellite navigation systems [14]. The precision improvement is realized by the dissemination of wide range differential corrections while the safety is ensured by the provision of integrity information. The information about the navigation system failure must be detected within $6 \mathrm{~s}$; therefore, the drop of the message reception probability is critical. The probability of a reception of the message depends, inter alia, on a signal to noise ratio and a bit rate, which is 10 times higher than the GPS message bit rate. Thus, the SBAS receiver requires a much higher signal to noise ratio for proper reception of the navigation messages than the GPS [14], so the aforementioned SNR drop can surely endanger the integrity of the satellite navigation system. Even though the observations of a degradation of the SNR of GPS and SBAS caused by the solar bursts were documented in [6], the detailed information about the impact of observed phenomena onto the system performance is however missing and should be an object of a future research.

\subsection{The Motivation for Modernization of Solar Radio Spectrography Instruments}

The solar radio emission in $\mathrm{L}$ band is in the research focus of the Astronomical Institute of the Czech Academy of Science in Ondrejov [15] for many years. The current scientific instrumentation RT5 (Figure 1) is more than 25 years old. Its performance and architecture reflect the time of development. The spectrograph based on an analog technology is in the operation since 1993 without significant design changes. Its performance like a resolution in time and frequency as well as its dynamic range and linearity is currently below the current requirements demanded by both the advance in technology and the increasing level of knowledge in the solar plasma physics. 


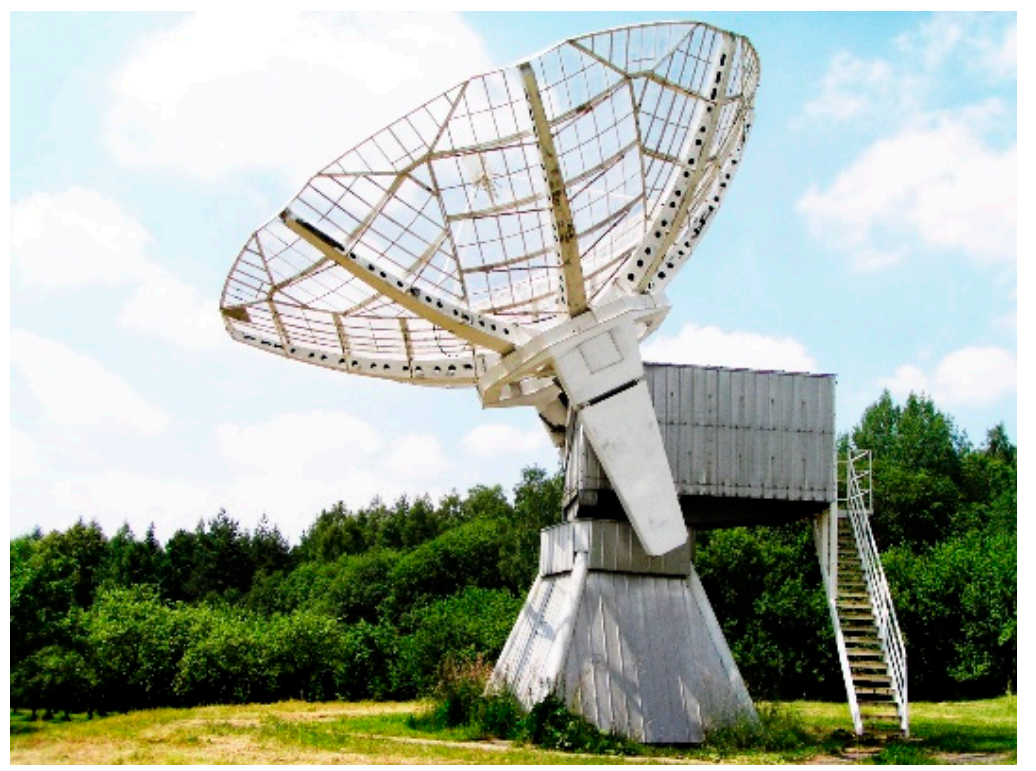

Figure 1. Antenna of Ondrejov RT5 solar radio spectrograph telescope with $9.5 \mathrm{~m}$ antenna diameter and operating frequency range $0.8-2.0 \mathrm{GHz}$.

The aim of the modernization project is to develop a modern but cost-effective instrument comparable or superior to the equipment of other observatories. The modern telescopes are usually designed as wideband radio receivers with a digital signal processing $[16,17]$ and their technology enables us to estimate the frequency spectrum in parallel for all frequency bins in comparison with the analog technology that is based on a scanning principle.

The analog spectrum estimation is usually based on the application of a super heterodyne receiver that sequentially scans the frequency spectrum channel-by-channel and measures the signal power [18]. The modern digital methods are based on an application of the discrete Fourier transform (DFT) or computationally optimized fast Fourier transform (FFT) [19-21]. The common method for a consequent spectrum estimation based on the Fourier transform is a short-time Fourier transform (STFT) [22,23].

There are several possible ways how to implement such spectrum computation algorithms. Therefore, the critical design analysis has to be made to choose an optimal design not only to fulfill the system requirements but also to respect implementation issues and realization costs.

The observations and records from the modernized spectrograph will be used both for a study of solar processes and for an investigation of the impact of a solar radio emission onto the current and future communication and radio navigation systems in the frame of several research projects.

\section{Design Concepts Analysis}

\subsection{Technical Requirements}

The technical requirements on the solar radio spectrograph were defined on the base of the long-term experiments with the current spectrograph with respect to the parameters of the similar instruments $[17,18,24,25]$.

The main attention during the conceptual design was paid to a high sensitivity and a wide dynamic range. The secondary design parameters superseding the parameters of the current analog instrument [15] were:

- $\quad$ Processing frequency range min. $1 / 2 \mathrm{GHz}$.

- Frequency bins spacing $<1 \mathrm{MHz}$.

- $\quad$ Time resolution $\leq 1 \mathrm{~ms}$. 
The high sensitivity requirement means that the noise floor of the telescope is below the quiet Sun radiation (QSR) level to be able to recognize its variation. The spectral flux density of the quiet Sun varies from 50 to $300 \mathrm{SFU}$ while the maximal observed solar burst flux is up to 1,000,000 SFU [11].

The classical approach of the radio spectrograph design is based on a super heterodyne receiver that sequentially scans individual frequency bins. The number $N$ of the investigated bins can be expressed as:

$$
N=\frac{f_{\max }-f_{\min }}{\Delta f}
$$

where $f_{\max }-f_{\min }$ is a frequency range and $\Delta f$ is a frequency spectra bins spacing. The processing time $t_{p}$ of an individual bin is:

$$
t_{p}=\frac{t_{r}}{N}-t_{t}
$$

where $t_{r}$ is a time resolution and $t_{t}$ is a time for returning to the next frequency bin. Let us note, that $t_{t}$ consists of a time for local oscillator retuning and a time for the removing of the transmission of the receiver filters. The time of measurement (i.e., the averaging in each frequency bin) is very short and thus the sensitivity (noise suppression) is poor.

A modern approach oriented on the digital processing is based on the digitalization of the whole signal in early stages of the reception chain and frequency spectrum is then estimated by FFT. The results are further smoothed (a Bartlett's approach [26]) with the aim of reduction of the power spectra estimation variance. The averaged samples of the power spectral density $C_{k}^{\prime}$ are expressed by:

$$
C_{k}^{\prime}=\frac{1}{M} \sum_{m=0}^{M-1} C_{k, m},
$$

where $C_{k, m}$ is a discrete power spectral density calculated from the $m$-th vector of input samples and $M$ is a number of averaged consecutive vectors of the signal samples.

The variance of the frequency spectra estimation is reduced $M$ times.

$$
\operatorname{var}\left[C_{k}^{\prime}\right]=\frac{1}{M} \operatorname{var}\left[C_{k, m}\right]
$$

Since $M \geq N$ and number of frequency bins is several hundreds or thousands, the typical improvement of SNR in the modern "parallel" approach is about $20 \mathrm{~dB}$ or more in comparison to the "serial scanning" approach in the past.

\subsection{Receiver Hardware Concept}

There are several existing projects of analog scanning and digital FFT based solar spectrographs [18, $24,25,27,28]$, where usually prevails an approach of the reception, detection, and sensing of signal in the analog domain with a final conversion of the detector output to digital data for the storage and viewing. On the other hand, a modern digital spectrograph is de facto a wideband receiver equipped with the FFT processor for the signal spectrum calculation from samples produced by an analog-to-digital converter (ADC) working in baseband or intermediate-frequency band output of receiver. As the spectrograph is a unique device, there are no usable application specific integrated circuits (ASICs) for its realization (apart from the extremely high costs for their development), so the digital processor is usually programmed to a field-programmable gate array (FPGA) or a digital signal processor (DSP) [24]. This architecture is often called a software radio or a software defined radio (SDR).

The preliminary design study identifies several critical problems:

1. Need of the high-speed analog-to-digital converters;

2. Cost of the high-performance FPGA; 
3. Software IP cores for FPGA for interfacing the ADC;

4. Challenging design of high selectivity wideband radio frequency (RF) filters or intermediate frequency (IF) filters.

The first design concept assumed to use a high-speed ADC with the several gigahertz sampling rate to be able to process a full $1 \mathrm{GHz}$ bandwidth in a single channel (Figure 2a). Such a choice would however lead to a very high cost of such ADC and a necessity to use a commercial proprietary IP (intellectual property) core for the interface between the ADC and the FPGA through JESD204B standard [29] that was also very expensive. The further cost analyses had shown that if the processing bandwidth would be divided into several sub-bands (a multi-channel approach, Figure $2 b$ ), the much cheaper ADCs with LVDS (low-voltage differential signaling) interface that does not need an expensive IP core can be used and thus the final cost of the instrument would be then significantly reduced. Let us note, that there is no need to process the working bandwidth in a single channel neither from signal processing nor scientific point of view. The cost saving brought by the multi-channel solution is so high that the total hardware cost of four channels is lower than the price of JESD204B IP core for the FPGA. Therefore, we decided to use the multi-channel concept in our modernization project.

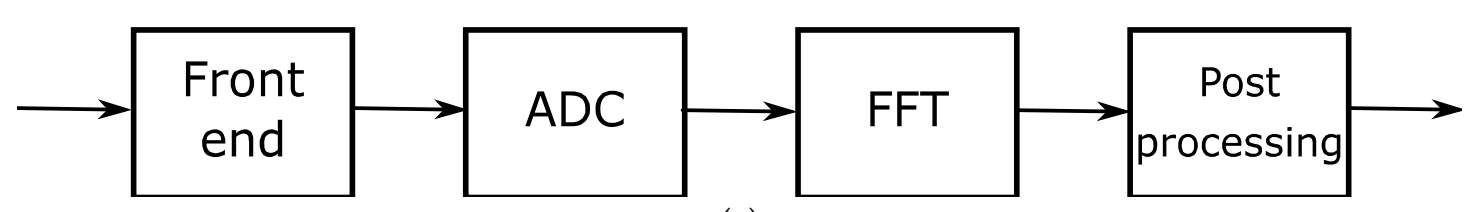

(a)

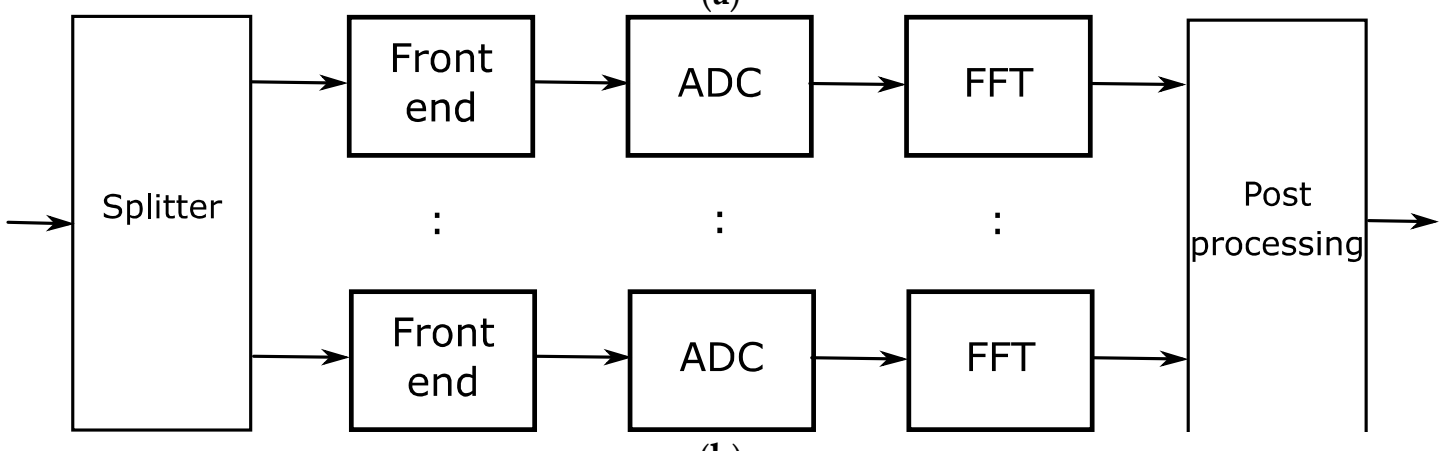

(b)

Figure 2. Spectrograph basic concept: (a) Single channel and (b) multi-channel.

The problem with a design and realization of high selectivity RF or IF filters, required in the front end for a suppression of the overlapping spectral components in case of the heterodyne concept, can be solved by a selection of the direct conversion receiver (Figure 3) that requires high selectivity low-pass filters instead of the band-pass ones. Let us note, that the low-pass filter with a bandwidth of several hundred megahertz can be realized as a lumped elements filter from standard SMD components while the band-pass IF filter of a super heterodyne receiver or the RF filter of a tuned radio receiver front end must be realized as a customized product based on the expensive ceramic resonator filter technology or on other special filter technology. The selectivity of the RF bandpass filter at the input of front end is not critical because it shall only additionally suppress the products outside the processed band 1-2 $\mathrm{GHz}$ and its realization is common for all channels. 


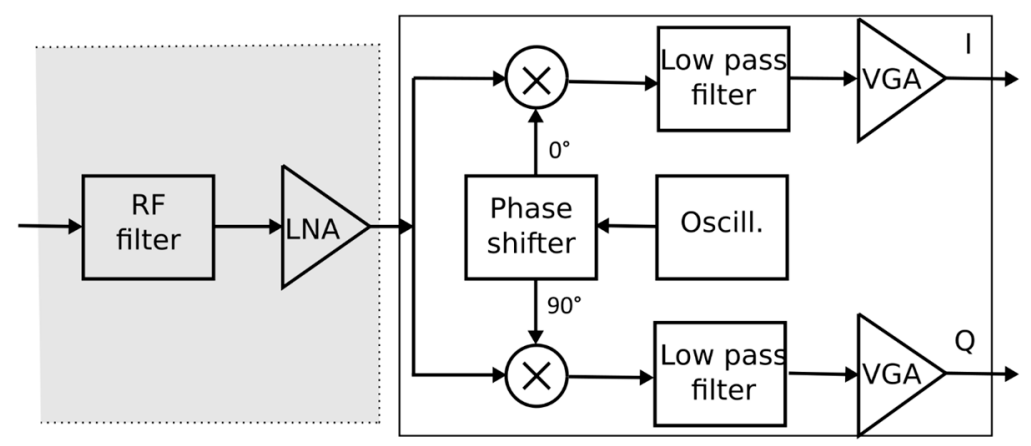

Figure 3. Front end realized by a direct conversion receiver with an optional radio frequency (RF) filter and LNA (low noise amplifier) at the input.

On the other hand, a direct conversion receiver [30-33] suffers from the following problems:

1. Problem with the semiconductor noise $1 / \mathrm{f}$.

2. DC offsets, and an interference of the local oscillator that oscillates in the middle of the processing frequency band.

3. Amplitude and phase unbalance that causes a crosstalk between upper and lower sidebands.

Fortunately, the first problem can be solved by the careful design of the level plan. The signal must be sufficiently amplified before the conversion to the baseband to avoid an influence of the $1 / \mathrm{f}$ noise. In our case, the power consumption of the receiver front end is not critical so using a wide dynamic range quadrature mixer with higher power consumption enables successful mixing of the high-level signal from the amplified input stage.

The problem with a DC offset can be solved by compensation feedback circuits, however paid by the distortion of the central frequency on which oscillates a local oscillator. The most radio systems count with this fact and use modulation schemas that are resistive to this distortion.

The solution to this problem in our design is to use a higher frequency resolution of the signal. The distorted frequency bin is ignored and replaced by an interpolated value.

The crosstalk between upper and lower sidebands caused by the receiver imperfection or insufficient suppression is a problem that can completely spoil the measurement or can bring unwanted effects in the spectrum. The crosstalk suppression is expressed as:

$$
B S(d B c)=-10 \log \left(\frac{1+A_{U}^{2}-2 A_{u} \cos \theta_{U}}{1+A_{U}^{2}+2 A_{u} \cos \theta_{U}}\right),
$$

where $A_{U}$ and $\theta_{U}$ are the amplitude and phase unbalance, respectively. A typical amplitude and phase unbalance of an uncompensated quadrature direct conversion mixer is about $0.5 \mathrm{~dB}$ and $1^{\circ}$. The crosstalk suppression is then about $30 \mathrm{~dB}$.

The higher suppression can be achieved by the mixer calibration typically with the help of the feedback circuits. The receiver front end must be equipped with a possibility to control the DC offset and gain of the baseband I and $Q$ branches and adjust the phase shift of the local oscillator signal. The properly designed compensation can increase the cross talk suppression up to $60 \mathrm{~dB}$ that is enough for the most applications.

The outputs of DC offset, amplitude, and phase imbalance detectors can be expressed:

$$
\begin{gathered}
d_{D C, I}=\sum_{n} I_{n}, d_{D C, Q}=\sum_{n} Q_{n}, \\
d_{\text {amp }}=\sum_{n} I_{n}^{2}-\sum_{n} Q_{n}^{2},
\end{gathered}
$$




$$
d_{p}=\frac{\sum_{n} I_{n} Q_{n}}{\sum_{n} I_{n}^{2}+\sum_{n} Q_{n}^{2}} .
$$

The values, produced by these detectors, are then used for a compensation of negative effects described above by controlling the oscillator block and variable gain amplifiers (VGA) in both I and Q branches.

The outputs of I and Q branches of the front-end receiver are then passed to dual-channel ADC and the samples are processed in a FPGA realizing the FFT computation of the full-bandwidth spectrum. The computed samples of the spectrum are passed through a standard interface to a connected computer or server for further processing, viewing, and storage. The FPGA can be also programmed to realize a detection of the receiver imperfections described above and provide the results to the control and setup processor for the compensation purposes.

To be able to simply combine measurements of several spectrograph channels, the measurements must be synchronized with 1 PPS (pulse per second) pulses. The source of such synchronization signal can be for example a 1 PPS output provided by a GPS receiver or an arbitrary frequency standard. If the pulses are not present, the spectrograph channels are switched to the free running mode. The measurement cycle is prolonged on to $1.1 \mathrm{~s}$. The complete block scheme of one channel is at Figure 4 .

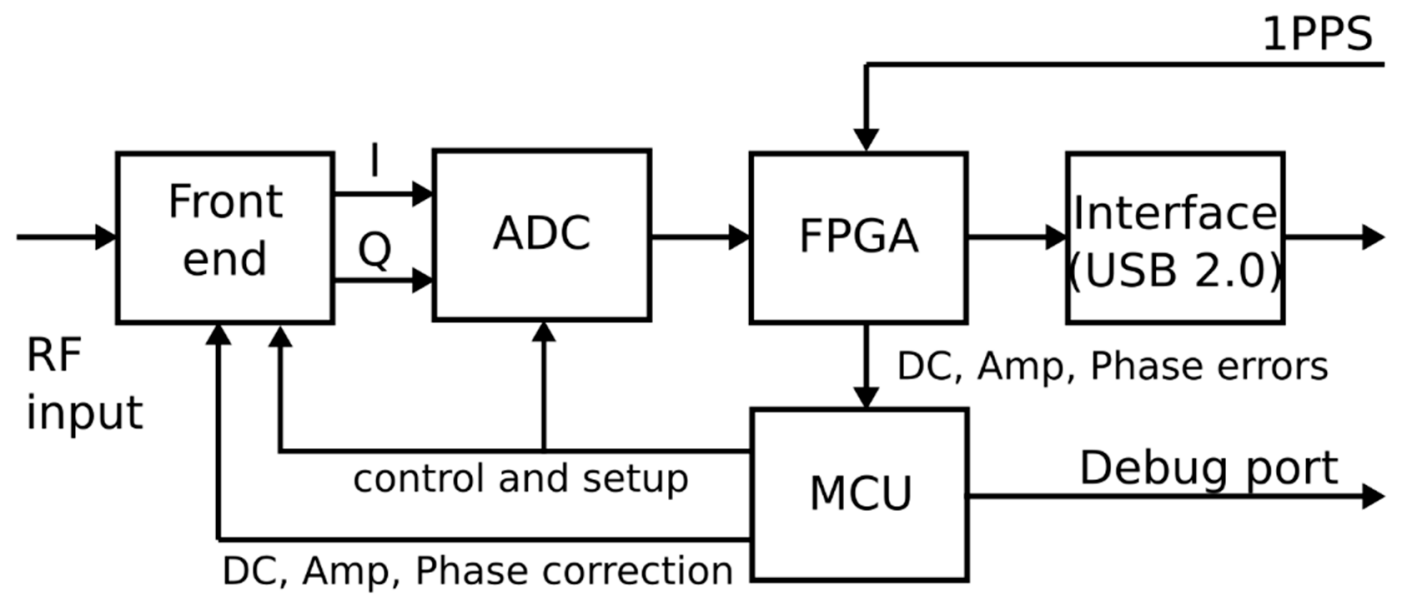

Figure 4. Spectrograph channel block diagram.

The communication between blocks and the output interface can be based on common standards (SPI, I2C, USB) according to parameters of chosen hardware components. The general control and setup are then usually provided by some kind of microcontroller unit (MCU).

\section{Implementation and Results}

\subsection{Spectrograph Hardware}

The described hardware concept was applied for a four-channel spectrograph in a frequency range of $1-2 \mathrm{GHz}$ (Figure 5) using the same RF band-pass filters and flat gain amplifiers both for the common RF input and for the front-end part of the channels. The RF filter is an of-the-shelf LTCC (low temperature cofired ceramics) filter (BFCG-162W+) with the pass band 950-2200 MHz, pass band insertion loss $1.8 \mathrm{~dB}$, and the stop band insertion loss better than $25 \mathrm{~dB}$. The flat gain amplifier is based on an off-the-shelf 40-4000 MHz RF gain block (TRF37C75) with $18 \mathrm{~dB}$ gain. These common blocks are placed together with an RF channel splitter, power supply modules, and a GPS module (for 1 PPS signal provision) to separate the board. Each spectrograph channel is then constructed as an independent block in its own case (Figure 6). The hardware parameters are summarized in Table 1. 


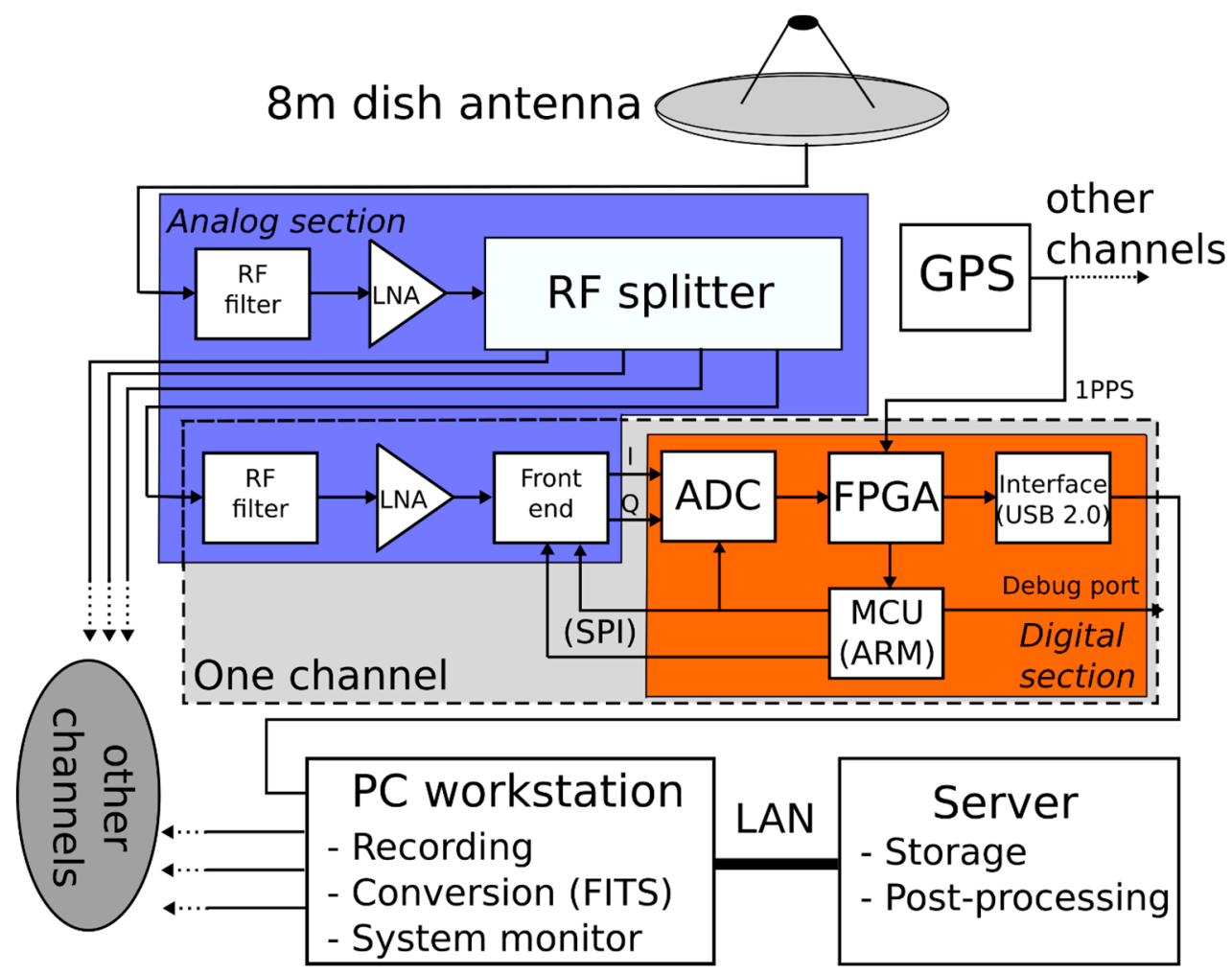

Figure 5. Realized spectrograph block scheme (only one channel depicted).

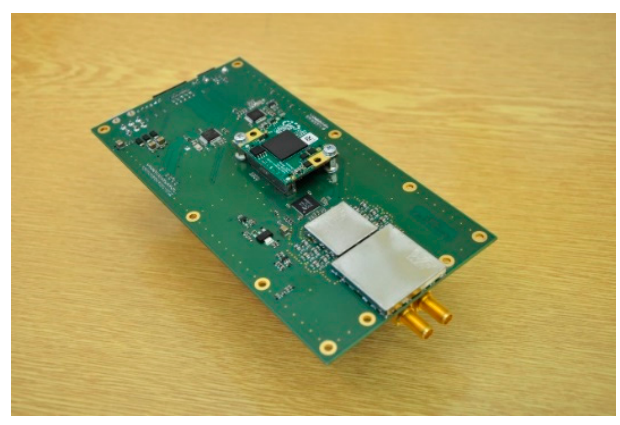

(a)

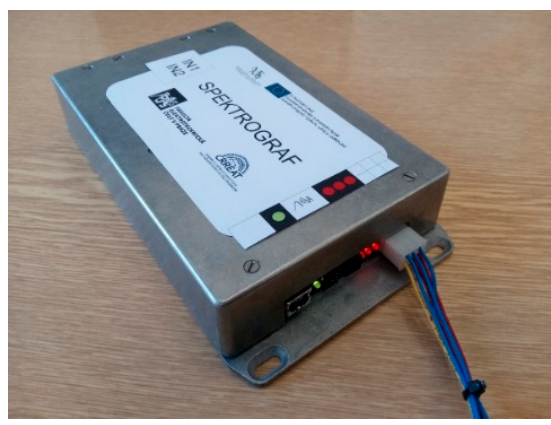

(b)

Figure 6. Spectrograph (single channel): (a) populated printable circuit board (b) housed to the aluminum case.

Table 1. Spectrograph hardware parameters (values for one channel).

\begin{tabular}{ccc}
\hline Parameter & Value & Note \\
\hline Usable bandwidth & $250 \mathrm{MHz}$ & \\
Operating range & $400 \mathrm{MHz} / 6 \mathrm{GHz}$ & (Center frequency) \\
Full Scale & $-25 /+5 \mathrm{dBm}$ & Programmable, step 1dB \\
Sampling rate & $310 \mathrm{MHz}$ & \\
ADC resolution & $2 \times 14 \mathrm{bits}$ & IQ sampling \\
Baseband filters & 6th order Cauer, symmetrical & \\
Filter pass-band & $0 / 125 \mathrm{MHz}, 0.5 \mathrm{~dB}$ ripple & \\
Stop-band & $\geq 155 \mathrm{MHz}$, Attenuation $\geq 50 \mathrm{~dB}$ & \\
Data interface & USB 2.0 device & Free running mode 1.1s if 1 PPS signal is not present \\
Synchronization & 1 PPS &
\end{tabular}

\subsection{FPGA Signal Processor Content}

The receiver is equipped with a medium rate FPGA Artix7 by Xilinx (San Jose, CA, USA). The block diagram of the FPGA configuration in Figure 7 demonstrates a sequence of processing operations. 
In order to meet the timing constraints, one second of a sampled signal data stream is separated in the splitter block to two consecutive half sized sub-blocks that are put into two parallel branches. The FFT operation with length 1024 is therefore calculated only with a half of processed data block and can work on a half of the sampling frequency, i.e., $155 \mathrm{MHz}$ (details in Table 2).

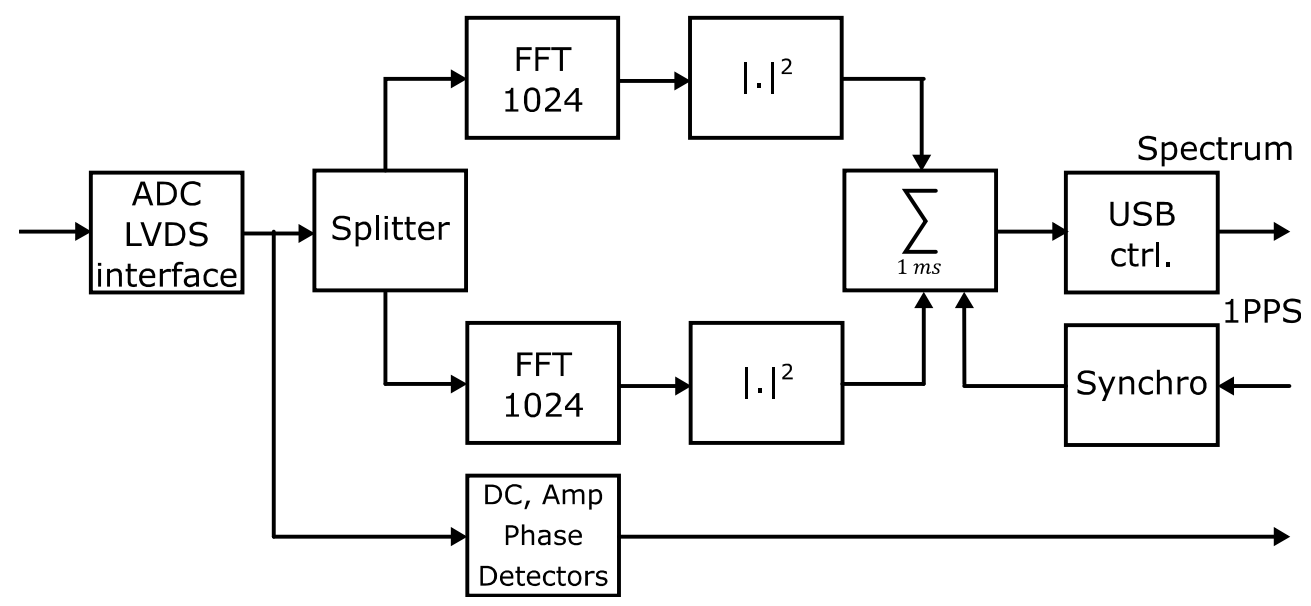

Figure 7. Field-programmable gate array (FPGA) block diagram.

Table 2. Signal processor settings.

\begin{tabular}{cc}
\hline Parameter & Value \\
\hline FFT length & 1024 \\
Frequency bin size & $302,704.375 \mathrm{~Hz}$ \\
Number of averaging $(\mathrm{M})$ & 300 \\
\hline
\end{tabular}

The next block executes a signal spectrum averaging of 300 FFT measurements with the averaging time of $1 \mathrm{~ms}$. This block is synchronized to the 1 PPS signal to be able to synchronize the spectrograph measurement onto an external time signal.

The measurement is then sent via the USB 2.0 interface to the server for storage and further processing. The data management software uses a standard FITS (flexible image transport system) format for data files [34].

The FPGA also realizes the detectors for a determination of the DC offset, amplitude imbalance, and phase imbalance. The compensation loops are closed through an adjacent ARM processor that controls through a SPI interface DC offsets, amplitude, and phase of the quadrature mixer. The other function of the ARM processor is a receiver general control and a setup of the front end and ADC.

\subsection{Testing}

During the spectrograph development and realization, the device was tested to verify the design parameters fulfillment. The test operation had started in the summer of 2018 at the Ondrejov astronomical observatory (Figure 8) with the aim of verification of the spectrograph performance and a comparison of its measurements with the current analog device. The developed spectrograph was tested with the backup $8 \mathrm{~m}$ dish antenna originated from the Second World War German radar while the current analog spectrograph is installed at the modern $9.5 \mathrm{~m}$ dish antenna that profits from the higher gain. The reason for such installation was to be able to test the new design parameters and operation setup without interruption of the current astronomical experiments. Due to a preliminary character of the tests, only one channel tuned to the center frequency $1525 \mathrm{MHz}$ was put in the operation. The test frequency band was chosen to cover the common satellite navigation systems' frequencies to be able to investigate the solar burst impacts on these systems as stated in the previous part of the paper. 


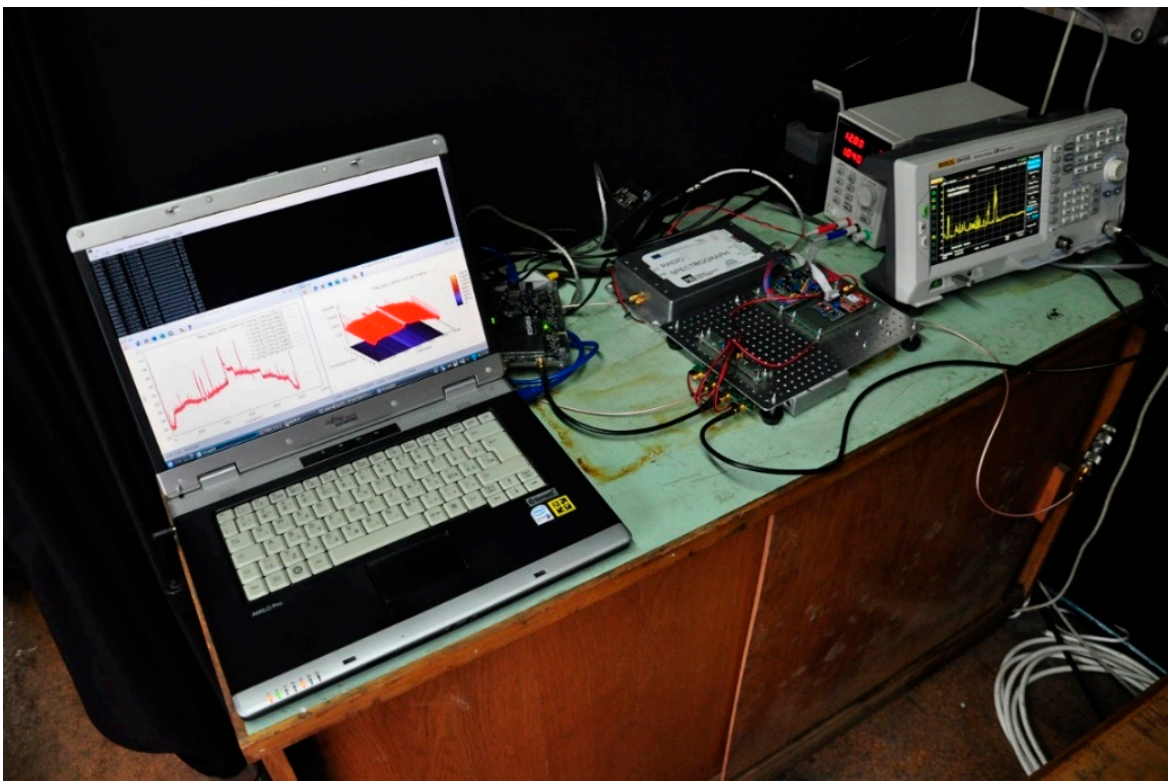

Figure 8. Spectrograph installation for tests at the Ondrejov observatory (one channel block and a common board with RF splitter, global positioning system (GPS) module, and power supply modules).

\subsection{Test Results}

During the early one-year test period only one observable event at 20 March 2019 was registered. The reason is that the Sun activity is at its long-term minimum. The spectrum registered by the current spectrograph is on Figure 9, while the measurements of the new digital spectrograph are on Figures 10-13. All spectrograms were depicted relative to reference time $\left(t_{r}=0 \mathrm{~s}\right)$ 11:11 UTC. Figures 10 and 11 represent the same spectrum part as a spectrogram to respect the style of Figure 9 and as a 3D spectrum waterfall plot. There was chosen an interval of $40 \mathrm{~s}$ that symmetrically covers the reference time for a demonstration of a modernized spectrograph data in full resolution. The datafile of the related interval represents a file of size $200 \mathrm{MB}$, therefore the long-time records are stored as one-second averages (Figure 13). They are also used for a coarse detection of solar events by an expert observer. The reference value of quiet Sun for all graphs is $100 \%$ (shown as $20 \mathrm{~dB}$ of relative power $\mathrm{P}_{\mathrm{r}}$ for new spectrograph images and as a ratio 1 for the old analog spectrograph). The drops of the spectrum on the sides of the evaluated band were caused by channel low-pass filter characteristics. This drop would be compensated by software processing during combination of overlapping adjacent channels.

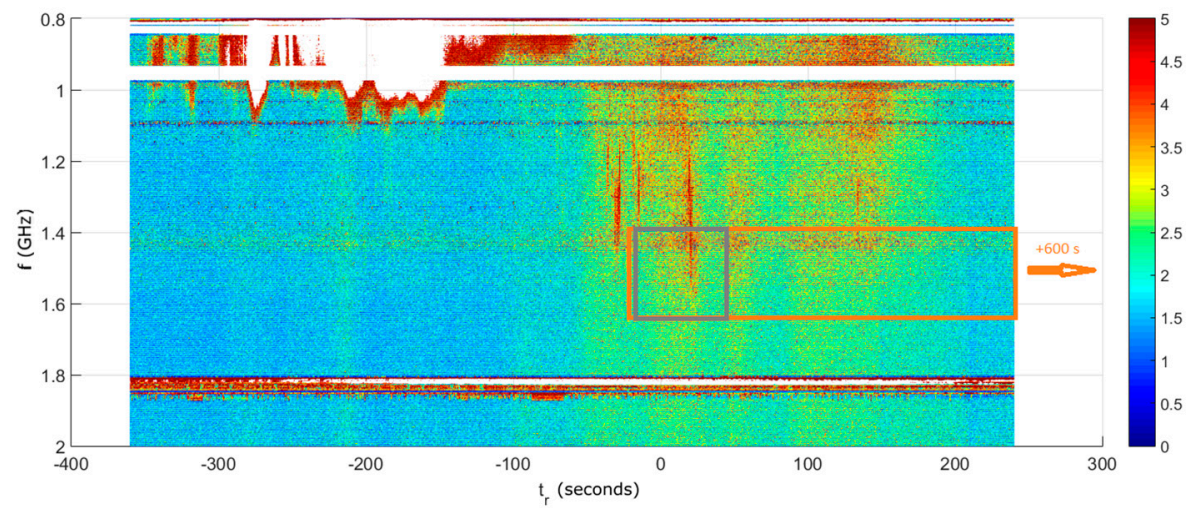

Figure 9. Spectrogram of solar radio burst, 20 March 2019, registered by current analog spectrograph. The rectangles show related portions of the spectrogram for a comparison with the new spectrograph, Figure 10-gray, Figure 13 (first $5 \mathrm{~min}$ )—orange). The colorbar values are expressed as relative values of the quiet Sun radiation (QSR) level (QSR = 1). 


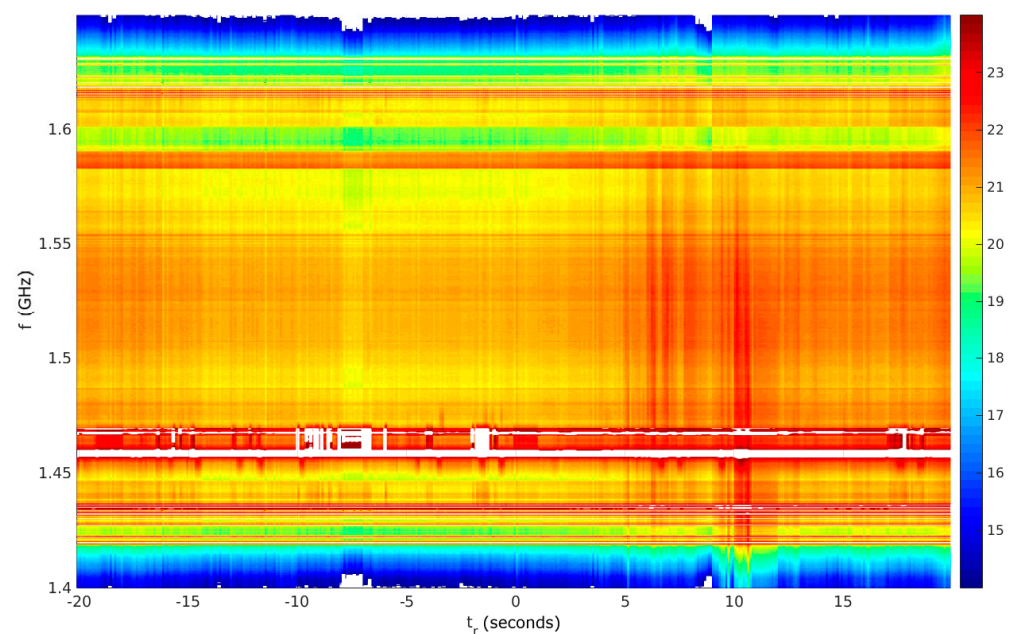

Figure 10. Spectrogram of solar radio burst, 20 March 2019, registered by the new spectrograph. The color scale is shown in $\mathrm{dB}$ relative to $\mathrm{QSR}=20 \mathrm{~dB}$.

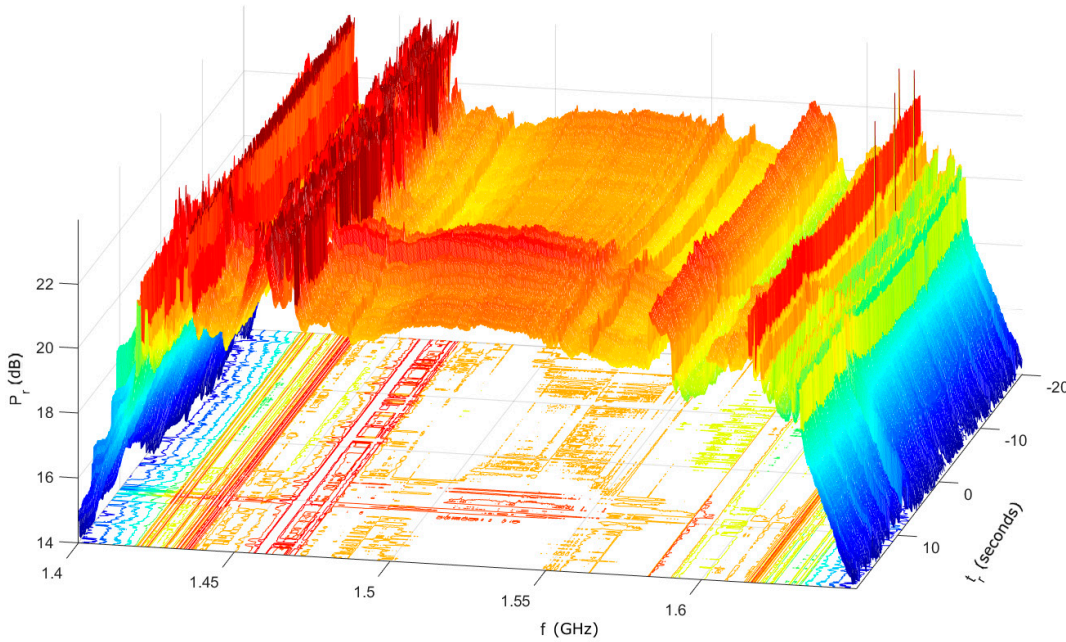

Figure 11. Solar radio burst (detailed 3D spectrum waterfall plot), March 20, 2019 registered by the new spectrograph (the time scale orientation is switched for the sake of better readability). 


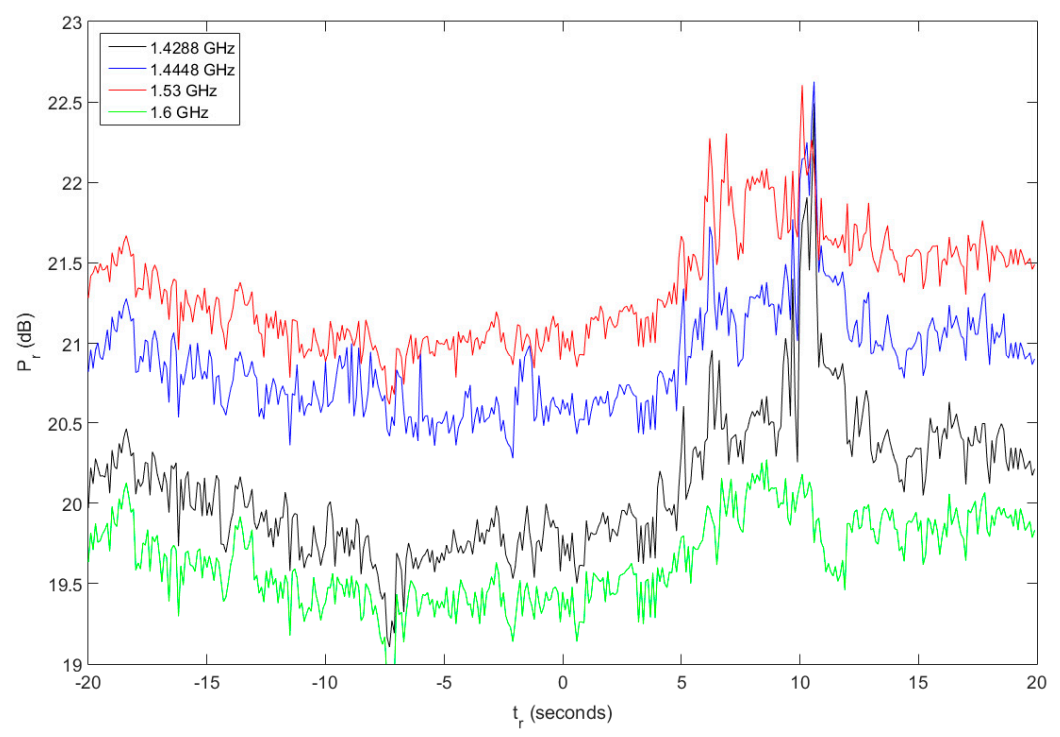

Figure 12. Solar radio burst (time record for selected frequencies), 20 March 2019 registered by the new spectrograph.

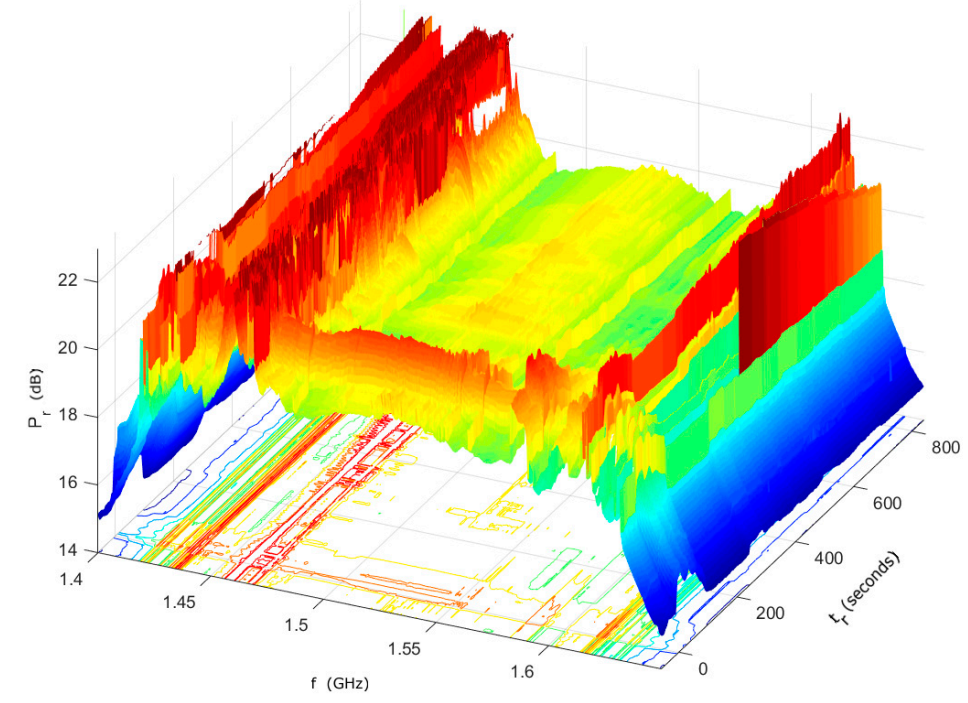

Figure 13. One-second averages (15 min total) of the 3D spectrum waterfall plot, 20 March 2019, reference time 11:11 UTC, registered by the new spectrograph.

To consider the system initial parameters, the measurements for a quiet Sun were executed as well (Figure 14) and compared to the values obtained by a system with the antenna pointed to the clear sky and during the night to determine a QSR-to-noise system ratio. 


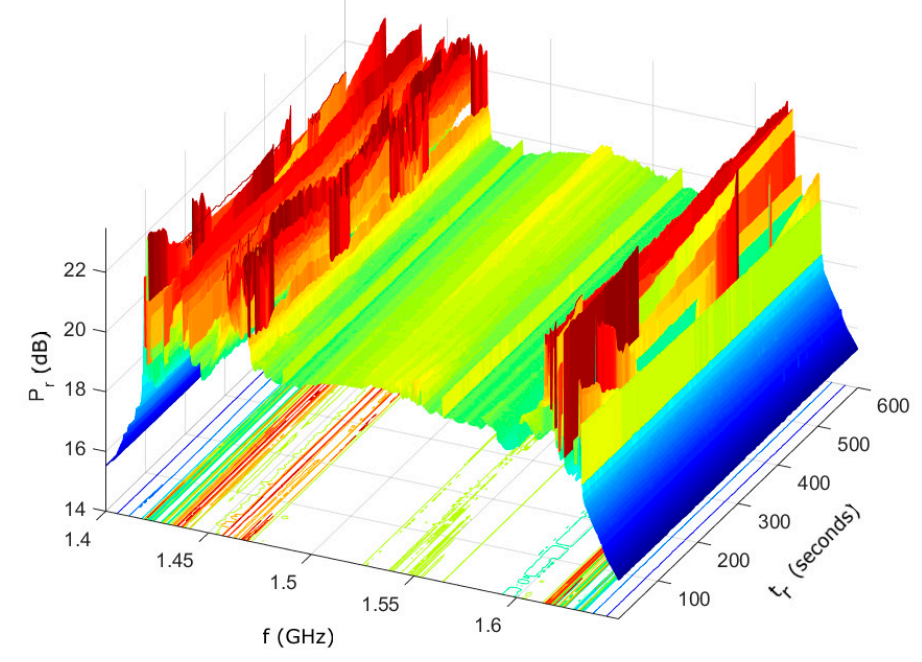

Figure 14. Quiet Sun case 3D spectrum waterfall plot obtained by the modernized spectrograph (a 10 min sample for relative time 11:40 UTC).

The observed difference of the signal level of about $3 \mathrm{~dB}$ for quiet Sun vs. clear sky does not sound very optimistic, but we have to take into account the non-optimal state of the used old antenna in comparison to the final installation. The most significant improvement can be achieved by the placement of a low noise amplifier directly to the antenna feed output as is in the case of the RT5 antenna. Nevertheless, we were still be able to observe quantitative changes of the spectrum level in comparison with the quiet Sun, therefore we can expect even better results for the full operation state. The expected values in the future are about $10 \%$ of the quiet Sun level, closer to the pure QSR to noise ratio obtained from the comparison of a QSR measurement with a night operation, where the ratios were $10 \mathrm{~dB}$ for single bin spikes of the spectrum and $15 \mathrm{~dB}$ for the average value of noise.

The frequency distribution of the noise level in the realized one channel band is mainly determined by the used low-pass filter characteristics as can be seen in 3D plots. Besides this impact, there were observed two spurs on frequency bins $1.436 \mathrm{GHz}$ and $1.615 \mathrm{GHz}$ caused by internal circuits of the receiver. These single-bin spurs will be excluded from the final data during the signal processing and substituted by an interpolated value from adjacent bins.

The testing measurements were also used to identify main sources of radio interference. The antenna placement is on the south-western slope of the hill in an elevation of about $510 \mathrm{~m}$. The nearby Ondrejov village is about $1 \mathrm{~km}$ from the observatory and with an elevation of about $450 \mathrm{~m}$, which helps to slightly reduce the impact of a possible close industrial interference. Main distant sources of the radio interference (considering the final full frequency range $1-2 \mathrm{GHz}$ ) can be a cellular phone network transmitter or a nearby mobile phone $(850 \mathrm{MHz}, 950 \mathrm{MHz}$, and $1800 \mathrm{MHz}$ band and higher harmonics of $850 \mathrm{MHz}$ band) and a digital terrestrial TV transmitter (higher harmonics of 562-706 and $714-858 \mathrm{MHz}$ bands). The impact of these interference sources can be suppressed by preventing the antenna pointing directly to the transmitter locations. The other source of the interference signal can be an ADS-B (automatic dependent surveillance-broadcast) signal at the frequency $1090 \mathrm{MHz}$. The signal is a product of an aircraft surveillance system transmitted in regular intervals during the flight and it can pose the notable source of interference because the location of a transmitter can be directly in the antenna beam due to the aircraft movement across the sky. Fortunately, such interference will be active only for a short time interval.

The notable interference was during the test operation observed at frequencies $1.458 \mathrm{GHz}$ with relative power $36 \mathrm{~dB}$ and $1.467 \mathrm{GHz}$ with relative power $27 \mathrm{~dB}$. Although the source of the interference was not identified, the observed levels are within designed dynamic range of the system and therefore do not cause a saturation of ADC. They will be digitally filtered in the processed signal data. Let us note that the RT5 spectrograph is more covered by the hill slope from interference sources and we can 
expect even better results in the future. Of course, the next interference analysis has to be made for the final installation to the location of current analog system.

\section{Discussion and Conclusions}

Even though the developed spectrograph used a lower gain old antenna during the tests, the obtained measurements carry out more details and a comparable background noise with respect to the analog spectrograph. The comparison of the parameters of both spectrographs is presented in the Table 3.

Table 3. Comparison of the parameters for both spectrographs.

\begin{tabular}{ccc}
\hline Parameter & RT5 Analog Spectrograph & Designed Digital Spectrograph \\
\hline Time resolution & $10 \mathrm{~ms}$ shared by 256 channels & $1 \mathrm{~ms}$ \\
Frequency resolution & $4 \mathrm{MHz}$ & $303 \mathrm{kHz}$ \\
ADC resolution & $12 \mathrm{bit}$ & $14 \mathrm{bit}$ \\
Dynamic range & $36 \mathrm{~dB}$ & $50 \mathrm{~dB}$ \\
QSR/noise ratio & $3.7 \mathrm{~dB}$ & $3-10 \mathrm{~dB}$ (impact of antenna) \\
\hline
\end{tabular}

The measurement of the current spectrograph is saved in a proprietary format while the measurement of the developed spectrograph is archived in the FITS format that allows further data analyses and easy sharing and manipulation. The indisputable advantage of the new spectrograph is a precise amplitude scale in contrast to the current one, which has its amplitude scale distorted because of the imperfect analog processing. Moreover, the higher time and frequency resolution of the new device enables a better identification and analysis of possible interference sources, such as microwave data links, mobile phone systems signals, air traffic radio navigation signals (e.g., ADS-B), terrestrial broadcast of digital television signals, etc.

The test of the L band solar spectrograph based on the modern digital signal processing at Ondrejov astronomical observatory confirmed the expected higher time and frequency resolution, and a high linearity of the amplitude measurement. The low processing noise that can be achieved by the massive averaging allowed by a parallel design (in contrast to the spectrum scanning approach of the current analog device) was however affected by the lower performance of the used antenna system and therefore the observed noise parameters persevered to stay at the original values. This problem is expected to be suppressed using the final installation antenna with better parameters. The crucial point was a proof of a validity of such design, which was accomplished according to the expectations.

As the test operation was evaluated as successful, the next step, expected in the end of 2019, will be an installation of the complete multi-channel system to the modern $10 \mathrm{~m}$ dish antenna.

The reasonable price of the spectrograph (approximately 2 kEuro per $250 \mathrm{MHz}$ channel) was achieved by splitting the operating frequency range to several synchronized $250 \mathrm{MHz}$ channels. This approach allows the use of moderately priced components and manufacture technologies without any negative impact on the developed instrument performance.

Author Contributions: Initiation of the spectrograph development and preliminary parameter specification, M.B.; development of the technical concept, P.K.; application of the spectrograph for investigation of the solar radio burst effect on the navigation and communication systems, P.K.; hardware development, FPGA programming and laboratory testing, P.K.; software for signal processing and the measurement format conversions and storage, P.P.; spectrograph installation P.P., P.K., M.B.; spectrograph operation, M.B.; preliminary measurement analyses, P.P.; writing—original draft, P.K.; writing—review and editing, P.P.; project administration P.K.

Funding: This research was founded by OP RDE, MEYS, Czech Republic under the project CRREAT, CZ.02.1.01/0.0/0.0/15_003/0000481 and by CTU project SGS18/142/OHK3/2T/13.

Conflicts of Interest: The authors declare no conflict of interest. The funders had no role in the design of the study; in the collection, analyses, or interpretation of data; in the writing of the manuscript, or in the decision to publish the results. 


\section{References}

1. NOAA/NWS Space Weather Impacts. Available online: https://www.swpc.noaa.gov/impacts (accessed on 15 June 2019).

2. Aschwanden, M.J.; Benz, A.O.; Dennis, B.R.; Schwartz, R.A. Solar Electron Beams Detected in Hard X-Rays and Radio Waves. Astrophys. J. 1995, 455, 347. [CrossRef]

3. Bárta, M.; Büchner, J.; Karlický, M.; Skála, J. Spontaneous Current-layer Fragmentation and Cascading Reconnection in Solar Flares. I. Model and Analysis. Astrophys. J. 2011, 737, 1-11. [CrossRef]

4. Carrano, C.S.; Bridgwood, C.T.; Groves, K.M. Impacts of the December 2006 solar radio bursts on the performance of GPS. Radio Sci. 2009, 44, 1-12. [CrossRef]

5. Muhammad, B.; Alberti, V.; Marassi, A.; Cianca, E.; Messerotti, M. Performance assessment of GPS receivers during the September 24, 2011 solar radio burst event. J. Space Weather Space Clim. 2015, 5, A32. [CrossRef]

6. Cerruti, A. Observed GPS and WAAS Signal-to-Noise Degradation Due to Solar Radio Bursts. In Proceedings of the 19th International Technical Meeting of the Satellite Division of The Institute of Navigation (ION GNSS 2006), Fort Worth, TX, USA, 26-29 September 2006; ION: Fort Worth, TX, USA, 2006; pp. 1369-1376.

7. Cerruti, A.P.; Kintner, P.M.; Gary, D.E.; Mannucci, A.J.; Meyer, R.F.; Doherty, P.; Coster, A.J. Effect of intense December 2006 solar radio bursts on GPS receivers. Space Weather 2008, 6, 1-10. [CrossRef]

8. Lanzerotti, L.; Gary, D.; Nita, G.; Thomson, D.; MacLennan, C. Noise in wireless systems from solar radio bursts. Adv. Space Res. 2005, 36, 2253-2257. [CrossRef]

9. Kennewell, J.A. Solar radio interference to satellite downlinks. In Proceedings of the 1989 Sixth International Conference on Antennas and Propagation, ICAP 89 (Conf. Publ. No.301), Coventry, UK, 4-7 April 1989; IET: Coventry, UK, 1989; pp. 334-339.

10. ITU Recommendation ITU-R P.372-13 (09/2016) Radio Noise; ITU: Geneva, Switzerland, 2016.

11. Nita, G.M.; Gary, D.E.; Lanzerotti, L.J.; Thomson, D.J. The Peak Flux Distribution of Solar Radio Bursts. Astrophys. J. 2002, 570, 423-438. [CrossRef]

12. O'Hanlon, B.; Kintner, P.M.S., Jr.; Kintner, P.M.; Gary, D.E. Global Positioning System and solar radio burst forensics. Radio Sci. 2009, 44, 1-6.

13. Joseph, A. What is $\mathrm{C} / \mathrm{N}_{0}$ and how it is calculated in a GNSS receiver. Inside GNSS 2010, 4, 20-29.

14. Enge, P. WAAS Messaging System: Data Rate, Capacity, and Forward Error Correction. Navigation 1997, 44, 63-76. [CrossRef]

15. Jiřička, K.; Karlický, M.; Kepka, O.; Tlamicha, A. Fast drift burst observations with the new Ondřejov radiospectrograph. Sol. Phys. 1993, 147, 203-206. [CrossRef]

16. Klein, B.; Krämer, I.; Hochgürtel, S.; Güsten, R.; Bell, A.; Meyer, K.; Chetik, V. Fast Fourier Transform Spectrometer. In Proceedings of the 20th International Symposium on Space Terahertz Technology, Charlottesville, VA, USA, 20-22 April 2009; ISSTT: Charlottesville, VA, USA, 2009; pp. 199-201.

17. Kallunki, J.; Uunila, M.; Monstein, C. Callisto radio spectrometer for observing the sun-Metsähovi Radio Observatory joins the worldwide observing network. IEEE Aerosp. Electron. Syst. Mag. 2013, 28, 5-9. [CrossRef]

18. Antar, Y.M.M.; Zawari, A.; Islam, M.T.; Anwar, R.; Hasbi, A.M.; Asillam, M.F.; Monstein, C. Callisto radio spectrometer construction at universiti kebangsaan malaysia [antennas and propagation around the world]. IEEE Antennas Propag. Mag. 2014, 56, 278-288. [CrossRef]

19. Dạbrowski, B.P.; Karlický, M.; Rudawy, P. Fourier Analysis of Radio Bursts Observed with Very High Time Resolution. Sol. Phys. 2015, 290, 169-180. [CrossRef]

20. Gough, P.T. A Fast Spectral Estimation Algorithm Based on the FFT. IEEE Trans. Signal Process. 1994, 42, 1317-1322. [CrossRef]

21. Müller, S.; Murk, A.; Monstein, C.; Kampfer, N. Intercomparison of Digital Fast Fourier Transform and Acoustooptical Spectrometers for Microwave Radiometry of the Atmosphere. IEEE Trans. Geosci. Remote. Sens. 2009, 47, 2233-2239. [CrossRef]

22. Kim, B.; Kong, S.-H.; Kim, S. Low Computational Enhancement of STFT-based Parameter Estimation. IEEE J. Sel. Top. Signal Process. 2015, 9, 1610-1619. [CrossRef]

23. Cabal-Yepez, E.; Garcia-Ramirez, A.G.; Romero-Troncoso, R.J.; Garcia-Perez, A.; Osornio-Rios, R.A. Reconfigurable Monitoring System for Time-Frequency Analysis on Industrial Equipment Through STFT and DWT. IEEE Trans. Ind. Inform. 2013, 9, 760-771. [CrossRef] 
24. Iwai, K.; Kubo, Y.; Ishibashi, H.; Naoi, T.; Harada, K.; Ema, K.; Hayashi, Y.; Chikahiro, Y. OCTAD-S: Digital fast Fourier transform spectrometers by FPGA. Earth Planets Space 2017, 69, 1-8. [CrossRef]

25. Ji, H.; Fu, Q.; Liu, Y.; Cheng, C.; Chen, Z.; Yan, Y.; Zheng, L.; Ning, Z.; Tan, C.; Lao, D.; et al. A Solar Radio Spectrometer at 5.2-7.6 GHz. Sol. Phys. 2003, 213, 359-366. [CrossRef]

26. Gismalla, E.H.; Alsusa, E. On the performance of energy detection using Bartlett's estimate for spectrum sensing in cognitive radio systems. IEEE Trans. Signal Process. 2012, 60, 3394-3404. [CrossRef]

27. Benz, A.O.; Isliker, H.; Miszkowicz, S.; Stehling, W.; Benz, A. A broadband spectrometer for decimetric and microwave radio bursts: First results. Sol. Phys. 1991, 133, 385-393. [CrossRef]

28. Benz, A.O.; Monstein, C.; Meyer, H. Callisto-A New Concept for Solar Radio Spectrometers. Sol. Phys. 2005, 226, 143-151. [CrossRef]

29. JEDEC. JEDEC Standard. Serial Interface for Data Converters. JESD204C (Revision of JESD204B.01 January 2012); JEDEC: Arlington, VA, USA, 2012; Available online: https://www.jedec.org/system/files/docs/JESD204C.pdf (accessed on 17 June 2019).

30. Horlin, F.; Bourdoux, A. Digital Compensation for Analog Front-Ends, 1st ed.; Wiley Blackwell: Hoboken, NJ, USA, 2008; ISBN 978-0-470-51708-6.

31. Gokceoglu, A.; Dikmese, S.; Valkama, M.; Renfors, M. Energy Detection under IQ Imbalance with Singleand Multi-Channel Direct-Conversion Receiver: Analysis and Mitigation. IEEE J. Sel. Areas Commun. 2014, 32, 411-424. [CrossRef]

32. Choi, M.; Choi, S. Performance analysis on the self-mixed interference cancellation in direct conversion receivers. IEEE Trans. Consum. Electron. 2013, 59, 310-315. [CrossRef]

33. Park, B.-K.; Yamada, S.; Lubecke, V. Measurement Method for Imbalance Factors in Direct-Conversion Quadrature Radar Systems. IEEE Microw. Wirel. Compon. Lett. 2007, 17, 403-405. [CrossRef]

34. Pence, W.D.; Chiappetti, L.; Stobie, E.; Page, C.G.; Shaw, R.A. Definition of the Flexible Image Transport System (FITS), version 3.0. Astron. Astrophys. 2010, 524, 1-40. [CrossRef]

(C) 2019 by the authors. Licensee MDPI, Basel, Switzerland. This article is an open access article distributed under the terms and conditions of the Creative Commons Attribution (CC BY) license (http://creativecommons.org/licenses/by/4.0/). 